\title{
FINITELY GENERATED SUBGROUPS OF AMALGAMATED FREE PRODUCTS AND HNN GROUPS
}

\author{
DANIEL E. COHEN
}

(Received 4 April 1975)

\begin{abstract}
The theory of groups acting on trees due to Bass and Serre (1969) is applied to simplify some results of Burns $(1972,1973)$ giving conditions under which an amalgamated free product or HNN extension has the properties that any finitely generated subgroup containing an infinite subnormal subgroup must have finite index and that the intersection of two finitely generated subgroups is finitely generated.
\end{abstract}

Burns $(1972,1973)$ gives sufficient conditions for an amalgamated free product $A{ }_{U} B$ or $H N N$ group $\left\langle A, x ; x^{-1} U x=V\right\rangle$ to have the following properties: any two finitely generated subgroups intersect in a finitely generated subgroup, and any finitely generated subgroup containing a (sufficiently large) subnormal subgroup has finite index. His conditions are on the position of $U$ in $A$, and include the case that $A$ is free and $U$ is cyclic generated by an element which is not a proper power. He observes that one can deduce from his results that a Fuchsian group has these properties.

In this paper we prove (and slightly extend) Burns's results using the Bass-Serre theory of groups acting on trees (Cohen (1974), Serre (1969)). To some extent this paper is propaganda for the view that the Bass-Serre theory leads to simpler proofs than more combinatorial methods (because the tree carries information that does not need to be mentioned explicitly). This paper is a sequel to Cohen (1974), to which the reader is referred for notation and many results.

\section{Burns subgroups}

The subgroup $U$ of the group $A$ is called a Burns subgroup if it has a left transversal $T$ with $1 \in T$ which satisfies the following two conditions:

(1) there is a finite subset $F$ of $U$ such that $U(T-\{1\}) \subseteq T F$; 
(2) for any finitely generated subgroup $H$ of $A$ and element $a \in A$ there is a finite subset $F_{1}$ of $U$ (depending on $H$ and $a$ ) such that $a H \subseteq T F_{1}(H \cap U)$.

These subgroups are introduced by Burns $(1972,1973)$ who calls them almost malnormal finitely involved, or AMFI, subgroups. Burns (1972) shows that when $A$ is free and $U$ is cyclic, generated by an element which is not a proper power, then $U$ is a Burns subgroup of $A$. Plainly any finite subgroup is a Burns subgroup, so the results and methods of this paper extend those of Cohen (1974).

(2) is plainly equivalent to:

( $\left.2^{\prime}\right)$ for any finitely generated subgroup $H$ of $A$ and element $a$ there is a finite subset $F_{2}$ of $A$ (depending on $H$ and $a$ ) such that $H a \subseteq$ $T F_{2}\left(a^{-1} \mathrm{Ha} \cap U\right)$.

(1) implies

(3) for any $a \notin U, U \cap a U a^{-1}$ is finite (which explains why $U$ is referred to as almost malnormal).

For we may assume $a \in T-\{1\}$. If $u=t v t^{-1}$, where $u, v \in U$ and $t \in T-\{1\}$, then $t v=u t \in T F$ shows $v \in F$, as required.

From (2) follows

(4) for any finitely generated subgroups $H, K$ of $A$, the intersection of a $(H, U)$ double coset and a $(K, U)$ double coset contains only finitely many ( $H \cap K, U)$ double cosets (see the remark in section 4 of Burns (1972)).

Replacing $\mathrm{H}, \mathrm{K}$ by $\mathrm{g}^{-1} \mathrm{Hg}, \mathrm{g}^{-1} \mathrm{Kg}$ if necessary, we need only consider $H U \cap K U$. Let $k=h u$, where $h \in H, k \in K$, and $u \in U$. There exist finite subsets $F_{1}, F_{2}$ of $U$ with $H \subseteq T F_{1}(H \cap U)$ and $K \subseteq T F_{2}(K \cap U)$. Then $u \in(H \cap U) F_{3}(K \cap U)$ where $F_{3}=F_{1}^{-1} F_{2}$ is a finite subset of $U$. Write $u=v c w$, where $v \in H \cap U, w \in K \cap U$, and $c \in F_{3}$. Then the $(H \cap K, U)$ double coset containing $k=h u$ will also contain $k w^{-1}=h v c$ which is an element of $K \cap \mathrm{Hc}$. Now $K \cap H c$ is a right $H \cap K$ coset. Hence every $(H \cap K, U)$ double coset contained in $H U \cap K U$ contains an element from one of finitely many right $H \cap K$ cosets, as needed.

LEMMA 1. Let $H$ be a finitely generated subgroup of $A$. Let $U$ be a subgroup of $A$ which is either a Burns subgroup or is finitely generated and satisfies (3) and (4). Then the intersection of $a(H, U)$ double coset and a $\left(H, a a^{-1}\right)$ double coset, where a£U, contains only finitely many right $H$ cosets.

Proof. As usual, we need only consider $\mathrm{HU} \cap \mathrm{HaUa}^{-1}$. Choose an element $h u=a v a^{-1}$ in some right $H$ coset, where $h \in H, u, v \in U$.

Let $U$ be a Burns subgroup of $A$. Then there is a finite subset $F_{1}$ of $U$ with $a^{-1} H \subseteq T F_{1}(H \cap U)$ and (writing $a^{-1}=t w$, with $\left.t \in T-\{1\}, w \in U\right)$ a finite subset $F_{2}$ of $U$ with $U a^{-1} \subseteq T F_{2}$. As $a^{-1} h u=v a^{-1} \in T F_{1}(H \cap U) u \cap T F_{2}$ we 
can write $u=k c$, where $k \in H \cap U$ and $c$ is in the finite set $F_{1}^{-1} F_{2}$. Then the right $H$ coset containing $h u=h k c$ also contains $c$, and so is one of finitely many cosets.

Suppose $U$ is finitely generated and satisfies (3) and (4). Then by (4) there is a finite subset $F_{1}$ of $U$ such that $h u=a v a^{-1} \in H U \cap a U a^{-1} U \subseteq$ $\left(H \cap a U a^{-1}\right) F_{1} U$. Write $h u=k c w$ where $c \in F_{1}, w \in U$, and $k \in H \cap a U a^{-1}$. The right $H$ coset containing $h u$ will also contain $c w=k^{-1} h u=k^{-1} a v a^{-1}=$ $a v^{\prime} a^{-1}$ for some $v^{\prime} \in U$. For given $c$, this element is in a unique left ( $U \cap a U a^{-1}$ ) coset, so by (3) there are only finitely many possibilities for this element.

Lemma 2. Let $U$ be a Burns subgroup of $A$, and let $V$ be a subgroup of $A$ such that there is a finite subset $F_{1}$ of $U$ with $V(T-\{1\}) \subseteq T F_{1}$ (for instance, $V \subseteq U)$. Let $H$ and $K$ be finitely generated subgroups of $A$ and $c, d \in A$. Then there is a finite subset $X$ of $U$ such that if $u \in U$ satisfies chu $=v d k$ for some $h \in H, k \in K, v \in V$ with $d k \notin U$ then $u \in(H \cap U) X(K \cap U)$.

Proof. There is a finite subset $F_{2}$ of $U$ such that $d k \in T F_{2}(K \cap U)$. As $d k \notin U$ we have $v d k \in T F_{1} F_{2}(K \cap U)$. There is also a finite subset $F_{3}$ of $U$ such that $c h \in T F_{3}(H \cap U)$. Then

$$
c h u=v d k \in T F_{3}(H \cap U) u \cap T F_{1} F_{2}(K \cap U),
$$

and so $F_{3}(H \cap U) u$ intersects $F_{1} F_{2}(K \cap U)$. Hence $u \in(H \cap U) F_{3}^{-1} F_{1} F_{2}(K \cap U)$.

\section{Bass-Serre theory}

If $G=A^{*}{ }_{v} B$ then $G$ acts without inversions on a tree $T$. There is an edge $e_{0}$ (the initial edge), whose stabiliser, stab $e_{0}$, is $U$. One vertex of $e_{0}$, the origin $O$, has stabiliser $A$, the other has stabiliser $B$. There is one orbit of edges and two of vertices, corresponding to $e_{0}$ and its two vertices. If $e$ is an edge with vertex $O$ then $e \in A e_{0}$.

If $G=\left\langle A, x ; x^{-1} U x=V\right\rangle$, an $H N N$ extension of $A$, then $G$ acts without inversions on a tree $T$. There is an edge $e_{0}$ (the initial edge) with stab $e_{0}=U$. One vertex of $e_{0}$, the origin $O$, has stabiliser $A$, the other is $x O$ with stabiliser $x A x^{-1}$. There is one orbit of edges and one of vertices. The edge $x^{-1} e_{0}$ has $O$ as a vertex and stab $x^{-1} e_{0}=x^{-1} U x=V$. If $e$ is an edge with vertex $O$ then either $e \in A e_{0}$ or $e \in A x^{-1} e_{0}$ but not both.

In either case if $e$ has vertex $O$ and $f$ is an edge with $f \in A e$, then any $h \in G$ with $f=h e$ must be in $A$. For let $f=a e$ with $a \in A$. Then $a^{-1} h e=e$ and as $G$ acts without inversions we must have $a^{-1} h O=O$, whence $a^{-1} h \in A$. For the 
same reason, if $e$ is an edge with vertex $P$ and $h e=e_{0}$ with $h P=O$ for some $h \in G$, then any $g$ with $g e=e_{0}$ has $g P=O$.

In either case we call an edge special if the following two conditions hold:

(i) the initial vertex of $e$ (orienting outwards from $O$ ) is in $G O$, but is not $O$;

(ii) if $P$ is the initial vertex of $e$, and $e^{\prime}$ the unique edge ending at $P$, then $e \in(\operatorname{stab} P) e^{\prime}$.

Note that if $P \neq O$, (i) always holds in the HNN case, while (ii) always holds for the amalgamated free product.

Lemma 3 below is essentially the same as Lemma 2.6 of Burns (1973), a reversing $H$-orbit containing a special edge being exactly what Burns calls a double-ended coset. For the definition and significance of reversing orbits see Cohen (1974). The reader is advised to draw parts of the relevant trees to help visualise the proofs of Lemma 3 and later results.

LemMA 3. Let $G$ be either an amalgamated free product or an HNN group, $T$ the tree described above on which $G$ acts. Let $H$ be a subgroup of $G$. Then $H$ has only finitely many reversing orbits provided only finitely many of its reversing orbits contain a special edge.

Proof. If $G$ is an amalgamated free product then any reversing $H$-orbit contains an edge starting at a vertex of $G O$, which is special unless it starts at $O$. Suppose a reversing $H$-orbit contains an edge $e_{1}$ starting at $O$, and let $h$ be negative for $e_{1}$. Then if $e \neq e_{1}$ is an edge starting at $O, H e$ contains $h e$, which is special since $h e_{1}$ is the only edge ending at $h O$. Hence every reversing $H$-orbit except perhaps $\mathrm{He}_{1}$ contains a special edge.

Now let $G$ be an HNN group. We first show that only finitely many reversing $H$-orbits contain an edge $e$ starting at $P \neq O$ (for some $P$ ) and such that another edge $f$ starting at $P$ is also in a reversing $H$-orbit.

For either $f \in(\operatorname{stab} P) e$ or $e \in(\operatorname{stab} P) e^{\prime}$ or $f \in(\operatorname{stab} P) e^{\prime}$, since there are only two $G$-orbits of edges at each point. In the first case choose $h \in H$ so that $h f$ ends at $h P$ (by choosing $h$ negative for $f$ ). As the edge he lies in (stab $h P$ ) $h f$, it is special, and so $e$ lies on one of finitely many orbits. The same holds in the second case, as $e$ is special.

In the third case $f$, being special, lies in one of finitely many orbits, hence so does $P$ (which is a vertex of $f$ ). In each orbit choose a vertex $P_{i} \neq O$ and (if possible) choose an edge $e_{i}$ starting at $P_{i}$ with $e_{i}$ in a reversing orbit and $e_{i} \notin\left(\mathrm{stab} P_{i}\right) e_{i}^{\prime}$, where $e_{i}^{\prime}$ is the edge ending at $P_{i}$. Let $P_{i}=h P$. Then either $h e=e_{i}^{\prime}$ or $h e$ starts at $P_{i}$ and lies in either (stab $\left.P_{i}\right) e_{i}^{\prime}$ or (stab $\left.P_{i}\right) e_{i}$ (for he is in a reversing orbit, so if $h e \notin$ (stab $\left.P_{i}\right) e_{i}^{\prime}$ an edge $e_{i}$ exists and every edge with vertex $P_{i}$ is in the (stab $\left.P_{i}\right)$-orbit of either $e_{i}$ or $e_{i}^{\prime}$ ). Thus either $h e=e_{i}^{\prime}$ or $h e$ is 
special or (as in the first case of the previous paragraph) he can be shown to be in one of finitely many orbits.

Also only finitely many reversing orbits contain an edge starting at $O$. For let $e_{1}, e_{2}$, and $e$ start at $O$ and lie in reversing orbits. Let $h$ be negative for $e_{2}$. Then $h e_{2}$ ends at $h O$, so $h O \neq O$, and both he and he start at $h O$. Hence he is one of the edges previously considered.

Choosing (at most) two suitable edges from each of the orbits we have considered, we obtain a finite set $X$ such that if $e$ is in such an orbit there is an $h$ positive for $e$ with $h e \in X$. Let $Y$ be the finite set consisting of all edges in the irreducible paths from $O$ to the edges of $X$. We show that any reversing orbit meets $Y$.

Note that if $e$ is in a reversing orbit either $e \in H X$ or there is an edge $f$, starting where $e$ ends, in a reversing orbit. For let $e$ end at $Q$. Choose $h$ negative for $e$, so that he starts at $h Q$. If $h Q=O$ then $h e \in H X$. Otherwise take $f$ to be the edge such that $h f$ ends at $h Q$.

Hence if $e_{1}$ is in a reversing orbit there is either an infinite sequence of edges $e_{1}, e_{2}, \cdots$, each in a reversing orbit, none in $H X$, with $e_{i}$ starting at $P_{i-1}$ and ending at $P_{i}$ for all $i$, or else there is a finite sequence of edges $e_{1}, \cdots, e_{n}$, each in a reversing orbit, with $e_{i}$ starting at $P_{i-1}$ and ending at $P_{i}$, with $e_{n} \in H X$.

In the first case choose $h$ so that $h e_{1}$ ends at $h P_{o}$. We cannot have $h e_{i}$ ending at $h P_{i-1}$ for all $i$, as this would require the path from $O$ to $h P_{o}$ to contain $h e_{i}$ for all $i$. Hence we can choose $r$ so that $h e_{r}$ ends at $h P_{r-1}$ but $h e_{r+1}$ does not end at $h P_{r}$. Then both $h e_{r}$ and $h e_{r+1}$ start at $h P_{r}$. By definition this gives $e_{r} \in H X$. This contradicts the definition of the sequence, so this case does not occur.

In the second case take $n$ minimal. Choose $h$ with $h e_{n} \in X$ and $h e_{n}$ ending at $h P_{n}$. If $h e_{i}$ ends at $h P_{i}$ for all $i$ the definition of $Y$ (since we are orienting outwards from $O$ ) gives $h e_{1} \in Y$. If not we can choose $r$ so that $h e_{r}$ ends at $h P_{r}$ while $h e_{r-1}$ ends at $h P_{r-2}$. Then both $h e_{r}$ and $h e_{r-1}$ start at $h P_{r-1}$. By definition of $X$ this gives $h e_{r-1} \in H X$. As this contradicts the minimality of $n$, this case cannot occur. Hence we must have $h e_{1} \in Y$, so $e_{1} \in H Y$, as required.

\section{The main theorems}

In the theorems below we consider a group $A$ and a Burns subgroup $U$. It is easy to see that we need only require the conclusions of Lemma 1 (or Lemma 2 ) to hold. Also we require certain subgroups of $U$ to be finitely generated. The easiest way to ensure this is to require that all subgroups of $U$ are finitely generated (the conditions stated require us to look outside $U$ ). 
THEOREM 1. Let $U$ be a Burns subgroup of $A$ and let $G$ be either $A{ }^{*} B$ or an $H N N$ group $\left\langle A, x ; U^{x}=V\right\rangle$. Let $H$ be a finitely generated subgroup of $G$ containing an infinite subnormal subgroup of $G$. If $H^{B} \cap U$ is infinitely generated for all $g \in G$ and $U \neq A$ (and $U \neq B$ also, in the first case) then $H$ has finite index in $G$.

Proof. We first show that any subnormal subgroup $N$ of $G$ contained in a conjugate of $A$ or $B$ must be finite. For it is easy to see that if $A_{1}$ is a subgroup of $A$ its normaliser will lie in $A$ unless it has a conjugate in $U$ or $V$. Hence we may assume $N \subseteq U$. Now $N$ is also subnormal in $A$. But any infinite subgroup of $U$ has $A$-normaliser in $U$, since $U \cap U^{a}$ is finite for $a \notin U$. Hence $N$ must be finite.

Theorem 8 of Cohen (1974) now tells us that there are only finitely many $(H, U)$ double cosets. For the same reason there are only finitely many $\left(H^{a}, U\right)$ double cosets, and so only finitely many $\left(H, a U a^{-1}\right)$ double cosets, where $a \in A-U$.

So it is enough to show that the intersection of any $(H, U)$ double coset and any $\left(H, a U a^{-1}\right)$ double coset contains only finitely many right $H$ cosets. As usual, it is enough to prove this for $\mathrm{HU} \cap \mathrm{HaHa}^{-1}$.

Any $H$ coset in $H U \cap \mathrm{HaUa}^{-1}$ can be represented by $h u=a v a^{-1}, h \in H$, $u, v \in U$. Plainly $h \in H \cap A$. But (Lemma 2 of Cohen (1974)) $H \cap A$ is finitely generated. Hence Lemma 1 gives the result.

THEOREM 2. Let $A$ and $B$ have the property that the intersection of any two finitely generated subgroups is finitely generated. Then the same property holds for $A *{ }_{U} B=G$ if $U$ is a Burns subgroup of $A$ and $H \cap U$ is finitely generated for all finitely generated subgroups $H$ of $G$.

THEOREM 3. Let $A$ have the above property, and let $G$ be the HNN group $\left\langle A, x: U^{x}=V\right\rangle$. Then $G$ has the same property if both $U$ and $V$ are Burns subgroups of $A$, and $H \cap U$ is finitely generated for all finitely generated subgroups $H$ of $G$.

Proofs. The two cases may be considered together, using the action of $G$ on the tree $T$ considered in Section 2. As in Theorem 7 of Cohen (1974), we know that $H \cap$ stab $P$ and $K \cap$ stab $P$ are finitely generated for any vertex $P$ of $T$, and that there are only finitely many reversing $H$-orbits and reversing $K$-orbits. As $H \cap K \cap$ stab $P$ will be finitely generated for any $P$, it will be enough to prove that there are only finitely many reversing $(H \cap K)$-orbits. Hence, by Lemma 3, it is enough to show that for any edge $f$ only finitely many reversing $(H \cap K)$-orbits in $H f \cap K f$ contain a special edge.

Take a special edge $e$ in a reversing $(H \cap K)$-orbit in $H f \cap K f$. We may 
assume there is a $g$ with $g O=P$ (the start of $e$ ) and $g e_{o}=e$. This is certainly possible for the amalgamated free product; for the HNN case if this does not hold we can find $g$ with $g O=P$ and $g x^{-1} e_{o}=e$. In the latter case we simply replace $e_{\mathrm{o}}$ and $U$ by $x^{-1} e_{0}$ and $V$ in the rest of the proof.

We may also assume (replacing $f$ by another edge, for instance $e$ itself, if necessary) that we can find $g_{1}$ with $g_{1} e_{o}=f$ and $g_{1} O$ the start of $f$. Then we may take $f=e_{o}$; if this does not hold we replace $A$ and $U$ by $A^{s_{1}}$ and $U^{B_{1}}$ but need make no other changes.

Since there are only finitely many reversing $H$-orbits, the edges which start at $O$ and lie in a reversing $H$-orbit will lie in finitely many $(H \cap A)$-orbits. Hence there is a finite subset $C$ of $A$ such that all these edges lie in $(H \cap A) C e_{\text {o }}$ if they are in $A e_{0}$. Similarly there is a finite subset $D$ of $A$ such that any edge in $A e_{0}$ which lies in a reversing $K$-orbit lies in $(K \cap A) D e_{0}$.

Now consider a special edge $e$ with $e=h e_{o}=k e_{o}$, where $h \in H, k \in K$, and let $e^{\prime}$ be the edge ending at $P$. As remarked at the beginning of Section 2 we must have $h O=P=k O$. Also $k=h u$ for some $u \in U$.

If $g$ is negative for $e$, then $g e$ ends at $g P$ and so $g e^{\prime}$ starts at $g P$, whence $g$ is negative for $e^{\prime}$. Hence $e^{\prime}$ is also in a reversing $(H \cap K)$-orbit. As $e$ is special we have $e^{\prime} \in(\operatorname{stab} P) e$, and so $h^{-1} e^{\prime} \in A e_{o}$. Hence $h^{-1} e^{\prime}=y c e_{o}$ for some $y \in H \cap A, c \in C$; similarly $k^{-1} e^{\prime}=z d e_{o}$ for some $z \in K \cap A, d \in D$.

Then $u z d e_{o}=y c e_{o}$, so that $u z d=y c w$ for some $w \in U$. This equation can be written as $c^{-1} y^{-1} u=w d^{-1} z^{-1}$. Also $z d \notin U$ as $k^{-1} e^{\prime} \neq e_{o}=k^{-1} e$. Consequently by Lemma 2 there is a finite subset $S$ of $U$ such that $u \in$ $(H \cap U) S(K \cap U)$ for all choices of special edge $e$ in $H_{0} \cap K e_{o}$.

Taking suitable $h^{\prime} \in h U, k^{\prime} \in k U$, we can write $e=h^{\prime} e_{o}=k^{\prime} e_{o}$ with $k^{\prime} \in h^{\prime} S$. If another edge $e_{1}$ corresponds to the same element of $S$, say $e_{1}=h^{\prime}{ }_{1} e_{o}=k^{\prime}{ }_{1} e_{o}$ then $e_{1}$ belongs to $(H \cap K) e$, since $k^{\prime}{ }_{1} k^{-1}=h^{\prime}{ }_{1} h^{-1} \in H \cap K$. Thus the special edges in $H e_{o} \cap K e_{o}$ lie in finitely many $(H \cap K)$-orbits, as required.

We conclude by discussing the group $G=\left\langle x, y ; y^{x}=y^{k}, k\right.$ an integer $\rangle$. Moldavanskii (1968) gave a short direct proof that the intersection of two finitely generated subgroups of $G$ is finitely generated. $G$ is an HNN group with $A=U=\langle y\rangle$ and $V=\left\langle y^{k}\right\rangle$, and so does not satisfy the hypotheses of Theorem 3 . We indicate how the previous proof can be modified.

Let $H$ and $K$ be finitely generated subgroups of $G$ and suppose $H \cap K \neq\{1\}$. Then we can find $g=x^{r} a x^{-s} \in H \cap K$ where $1 \neq a \in A$ and $r \geqq s \geqq 0$. If $r=s$ then $1 \neq g^{k^{\prime}} \in H \cap K \cap A$, since $g^{k^{\prime}}=x^{\prime} a^{k^{\prime}} x^{-r}=a$. Then any $(H, A)$ double coset contains only finitely many $H$ cosets, whence the intersection of a $(H, U)$ double coset and a $(K, U)$ double coset contains only finitely many $(H \cap K)$ cosets. If $r>s$, then $x^{n+r} e_{0} \in(H \cap K) x^{n+s} e_{0}$ for any $n \geqq 0$ and 
any reversing $(H \cap K)$ orbit will contain $x^{n} e_{o}$ for some $n$ with $0 \leqq n \leqq r$, so there are only finitely many such orbits.

\section{References}

R. G. Burns (1972), 'On the finitely generated subgroups of an amalgamated product of two groups', Trans Amer. Math. Soc. 169, 293-306.

R. G. Burns (1973), 'Finitely generated subgroups of HNN groups', Canad. J. Math. 25, 1103-1112.

D. E. Cohen (1974), 'Subgroups of HNN groups', J. Austral. Math. Soc. 17, 394-405.

D. I. Moldavanskii (1968), 'The intersection of finitely generated subgroups', (Russian), Sibirsk. Mat. Ž. 9, 1422-1426 (English translation in Siberian Math. J. 9, 1066-1069 (1969)).

J.-P. Serre (1969), Groupes Discretes (Lecture Notes, Collège de France).

Department of Pure Mathematics, Queen Mary College, London University, England. 